\section{Corona-Erkenntnisse für die Kreislaufwirtschaft}

Österreich verfügt über eine sehr hoch entwickelte Abfallwirtschaft und die Corona-Pandemie hat gezeigt, dass das System durchaus resilient ist. Das erhöhte Abfallaufkommen im Haushaltsbereich aufgrund der Ausgangssperren und Einschränkungen konnte für die BürgerInnen scheinbar problemlos bewältigt werden, die Abfallwirtschaft kam in den Medien im Zusammenhang mit Corona eigentlich kaum vor. Es gab keine Cluster, keine durch Müll verbreiteten Infektionen, lediglich die Altstoffsammelzentren waren für mehrere Wochen geschlossen und haben so mancher auf den ersten Blick gut getimten Entrümpelungsaktion einen Strich durch die Rechnung gemacht. Aber natürlich hat es abseits der öffentlichen Wahrnehmung einzelne Segmente der Abfallfallwirtschaft hart getroffen, wie z. B. das Textil- und das Kunststoffrecycling. Vor allem das Problem der Kunststoffrecycler zeigt ein prinzipielles Dilemma der derzeit geltenden Gesetzeslage und Praxis auf. Für Leichtverpackungen gibt es Mindestrecyclingquoten und diese werden durch die novellierte EU-Verpackungsrichtlinie mittelfristig sogar erhöht, was einige Anstrengungen für die heimischen Sammler und Recycler bedeutet. Die Corona-Krise hat aber unter anderem zwei für das Kunststoffrecycling problematische Verwerfungen mit sich gebracht: Zum einen ist der Ölpreis stark gefallen, was sich unmittelbar dämpfend auf den Preis von Primärkunststoffen auswirkt. Der Einsatz von Rezyklat wird damit weniger bis sogar unattraktiv. Zweitens ist der Konsum zurückgegangen und damit der Absatz von Kunststoffverpackungen bzw. -produkten generell.

Das alles hat zur Konsequenz, dass die Recycler ihr Rezyklat schwer am Markt unterbringen und die Zwischenlager voll und übervoll sind. Als eine Abhilfe der prekären Situation wird von der Recyclingwirtschaft eine Mindesteinsatzquote für Rezyklat ins Spiel gebracht. Dazu folgende Überlegung: Ein wesentlicher Unterschied zwischen Primärkunststoff und Rezyklat ist, dass der Rohstoff für Primärkunststoffe, das Rohöl, eingekauft wird und daher seine Menge für den Produzenten steuerbar ist. Der Rohstoff für Recycler stellt die sortierte Sammelware dar und diese ist mengenmäßig durch das Sammelaufkommen und indirekt durch die vorgegebenen Recyclingziele bestimmt. Das Rohstoffaufkommen ist also für den Recycler eine kaum beeinflussbare Größe. Weiters ist zu bedenken, dass der Konsum von Produkten eine zeitliche Verzögerung bewirkt, also eine Änderung des Konsums eine gewisse Zeit benötigt, bis diese in der Abfallwirtschaft ankommt. Während also die Primärproduzenten auf die sprichwörtliche Bremse steigen, kann es durchaus sein, dass es Monate dauert, bis dies in der Abfallwirtschaft ankommt.

Wenn man dies berücksichtigt, dann wird klar, dass eine Mindesteinsatzquote das Problem der Recycler auch nicht löst, weil fixe Größen in einem dynamischen System rasch aus ihrem optimalen Bereich fallen. Es müsste schon so etwas wie eine Abnahmegarantie durch die Produzenten sein. Dies widerspricht allerdings dem Prinzip einer freien Marktwirtschaft und wäre eher planwirtschaftlichem Handeln zuzuordnen. Und damit sind wir beim Punkt: Primär- und Sekundärrohstoffe haben unterschiedlich funktionierende Quellen und daher in einem freien Markt nicht die gleichen Voraussetzungen. In anderen Worten: Kreislauflaufwirtschaft und freie Marktwirtschaft vertragen sich nicht in ihren Reinformen. Um unseren derzeitigen (immer noch) linear geprägten Stoffhaushalt in eine ausgeprägte Kreislaufwirtschaft umzubauen, wird es massiver steuernder Eingriffe bedürfen. Ob dies möglich bzw. gesellschaftspolitisch erwünscht ist, muss sich erst zeigen. Es sollte zumindest vermehrt andiskutiert werden.

Die vorliegende Ausgabe der ÖWAW beinhaltet drei Originalarbeiten, die das breite Spektrum der Themen, mit denen sich die heutige Abfallwirtschaft und das Ressourcenmanagement befassen, sehr schön zeigen. Kornthaler und Bockreis untersuchen in ihrem Beitrag die Möglichkeiten und Herausforderungen für pfandbasierte Mehrweglösungen zur Verringerung des Abfallaufkommens im Con-

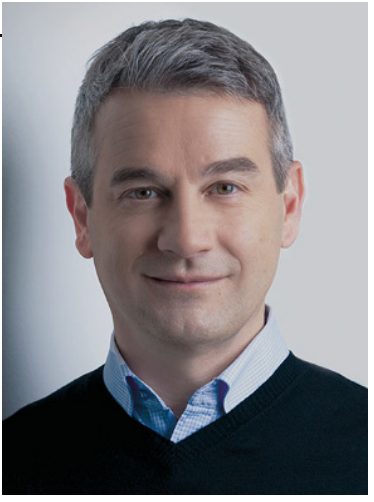

Univ.-Prof. DI Dr. Helmut Rechberger 
venience-Food-Bereich. Pomberger zeigt einen Weg auf, mit dem man die reale Recyclingfähigkeit von Verpackungen untersuchen und bewerten kann und dies auch lenkend bei der Ausgestaltung von Lizenzentgelten der Sammel- und Verwertungssystemen einsetzen könnte. Scherhaufer und Kollegen untersuchen Recyclingszenarien für den rasch wachsenden Sektor der Stromversorgung aus Windkraft- und Photovoltaikanlagen.

\section{Univ.-Prof. DI Dr. H. Rechberger ( $₫$ )}

Institut für Wassergüte und

Ressourcenmanagement, Technische

Universität Wien,

Karlsplatz 13,

1040 Wien, Österreich

helmut.rechberger@tuwien.ac.at 